\title{
Optical coherence tomography in progressive cone dystrophy
}

\author{
Jiri Zahlava, Jan Lestak, Ivan Karel
}

\begin{abstract}
Aim. The aim of the study was to analyse different clinical pictures in patients with progressive cone dystrophy (PCD), to compare these with the results of optical coherence tomography $(\mathrm{OCT})$ and to evaluate the benefits of this method for diagnosis.

Methods. The group consisted of 16 patients (32 eyes) with PCD. All patients were examined for visual acuity, colour sense and visual field. We performed biomicroscopic examination, photo-documentation, fluorescein angiography, electrophysiological tests and OCT.

Results. Using biomicroscopy and fluorescein angiography, we found changes in the retinal pigment epithelium ranging from barely detectable changes up to the typical bull's eye appearance. In all the eyes, OCT established statistically significant reduction in the thickness and structural changes in the neuroretina of the macula. Atrophy was evident especially in the outer nuclear layer, in the photoreceptor inner segment/outer segment junction and in the retinal pigment epithelium. Visual acuity was mainly dependent on the degree to which the continuity of the photoreceptor inner segment/outer segment junction layer was maintained. Eyes with better preserved neuroretinal structure in the fovea centralis had generally less reduced thickness of the retina and a better visual acuity.

Conclusion. OCT specifies the quantitative and qualitative changes in the macula and may contribute significantly to the diagnosis of the progressive cone dystrophy, particularly in the early stages of the disease which is difficult to diagnose.
\end{abstract}

Key words: progressive cone dystrophy, optical coherence tomography, photoreceptor inner segment/outer segment junction, outer nuclear layer

Received: August 14, 2012; Accepted: February 27, 2013; Available online: March 21, 2013

http://dx.doi.org/10.5507/bp.2013.017

Eye department of the Clinic JL, V Hurkach 1296, 15800 Prague 5, Czech Republic

Corresponding author: Jiri Zahlava, e-mail:jiri.zahlava@email.cz

\section{INTRODUCTION}

Cone dystrophy represents a heterogeneous group of macular dystrophies with variously expressed clinical manifestations and often non-characteristic or barely detectable changes in the macula which can be a source of diagnostic difficulties. The fairly rare cone dystrophy is classified into the congenital stationary form with inborn absence or functional disorder of the cones, and the progressive form ${ }^{1-7}$.

Progressive cone dystrophy (PCD) is inherited autosomally dominantly or recessively, or recessively linked to the $\mathrm{X}$ chromosome, however its incidence is often sporadic $^{1-12}$.

Visual acuity (VA) in the affected individuals is usually normal during childhood and only gradually deteriorates, often leading to legal blindness. Impaired VA is accompanied by a defective colour sense, visual field defects, photophobia ${ }^{1,2,4-10,13-15}$ and in some patients with affected rods also by nyctalopia, 3,6,7,9. Visual field defects include central scotomata ${ }^{1,3,6,9,12,13}$, annular scotomata around the centre ${ }^{1,3}$, diffuse reduction in sensitivity ${ }^{12}$ and rarely, peripheral scotomata ${ }^{1-3,6,9}$.

A finding in the macula may vary within the range of non-specific changes to the retinal pigment epithelium (RPE), to the characteristic RPE lesions of the bull's eye type ${ }^{1-7,9,13-15}$. Changes in the macula are rarely accompa- nied by the temporal disc pallor ${ }^{1,3,4,6,12-14}$, golden tapetal sheen $^{8,12}$, white dot-like lesions at the level of RPE mostly in the mid-periphery ${ }^{1,3}$, pigment shifts in the periphery $1,3,6,9,15$ and nystagmus ${ }^{2,4,6,9,11,14}$.

Electrophysiological examinations show clear reduction in or even elimination of the cone function with normal or reduced response of the rods. ERG changes precede the decline in subjective visual functions ${ }^{1-5,7-11,13-15}$. The forms of progressive dystrophy with functional deficiency limited to the photopic system are termed progressive cone dystrophy ${ }^{2,6}$. However, in most of the forms, a dysfunction of the rods is also likely to develop in the course of the condition, and these forms are then termed progressive cone-rod dystrophy ${ }^{6}$. These individuals often suffer from night blindness $\mathrm{s}^{3,6,7,9}$.

Optical coherence tomography (OCT) images a crosssection of retina of the posterior pole of the eye allowing to not only measure the retinal thickness but to evaluate its structural changes as well. While benefits of OCT in diagnosing most of the macular disorders have been assessed in numerous studies, only a few studies mention OCT findings in PCD. OCT findings in cone-rod dystrophy have only been described by Wolfing et al. ${ }^{16}$ in 1 patient, Lim et al. ${ }^{15}$ in 2 patients and Emfietzoglou et al. ${ }^{17}$ in 1 patient. To date, no OCT findings have been published in a larger group of patients with PCD.

The aim of the study was to analyse various clinical 
pictures in patients with PCD, compare these with the results of OCT and to evaluate the benefits of this method for diagnoses.

\section{MATERIALS AND METHODS}

The group consisted of 16 patients (32 eyes) with PCD, 7 males and 9 females age range 27-73 years, average age 42.6 years. All patients had good vision in childhood, vision began to deteriorate between year 10 and 69 of their lives, and had been progressing since. When first contacting our department their subjective problems had lasted for 0.5 to 29 years, 4.97 years on average. Five patients were members of two families. Patients in one of the families included a mother (the oldest member of the group), the son and daughter, while those of the other family were a brother and sister.

We examined all patients using the same protocol over 2 years. The distance visual acuity was measured with ETDRS optotypes with optimal correction, and expressed in decimal numbers. Colour sense was examined using the Lanthony's Desaturated 15-Hue test and the visual field was measured by a static perimeter - the threshold test. The eyegrounds, with a particular attention to the macula, were examined in artificial mydriasis using binocular indirect ophthalmoscopy, slit-lamp biomicroscopy with a contact lens, fluorescein angiography (FA) and electrophysiology. Findings in the macula were documented by colour photographs and images in red-free light.

OCT examinations of the macula were performed in artificial mydriasis by Stratus OCT (Version: 4.0.1., Carl Zeiss Meditec) using "scan acquisition protocols - macular thickness map" with special attention to fovea centralis. The images were $6 \mathrm{~mm}$ in length consisting of $512 \mathrm{~A}$ scans, each composed of 1024 axial data points. During the examination, the internal fixation was used and the location of the scan was controlled by the examining doctor.

Neuroretinal thickness in the macula, the distance between vitreoretinal interface and the anterior surface of the retinal pigment epithelial/choriocapillaris region were automatically determined by the OCT software and analyzed using data from all six linear scans.

Due to the inaccurate demarcation of the neuroretina in the pathologically changed fovea centralis by the Stratus OCT software, its thickness within the central region of the $0.5 \mathrm{~mm}$ radius was not evaluable. For this reason we measured the neuroretinal thickness in the centre of fovea centralis manually. The neuroretinal thickness within the annuli around the centre of fovea centralis, with radii of 0.5-1.5 mm (inner sector) and 1.5-3.0 mm (outer sector), was already evaluable by the OCT software.

In addition to the thickness, we also monitored structural changes of the neuroretina - images of the individual layers, their reflectivity and defects in continuity. Tomograms were evaluated from the false-colour scale as well as the grey scale which highlighted particularly the structure of the outer layers of the neuroretina.

For comparison of the neuroretinal thickness in the eyes with PCD and for the statistical evaluation, we drew up a set of 100 eyes in 100 individuals with normal function and normal biomicroscopical and OCT findings.

Statistical analysis was performed on SigmaStat software (SPSS Inc., Chicago, IL). The results are expressed as averages $\pm \mathrm{SD}$ (standard deviation). The comparison between the groups was performed by the Mann-Whitney Rank Sum Test. Statistical significance was assigned to $P<0.05$.

All the patients signed an informed consent to being included in the set and to the examinations performed.

\section{RESULTS}

All 32 eyes in the 16 patients had reduced VA, from 0.8 to 0.025 , and the average VA was $0.41 \pm 0.28$. Twenty four eyes $(75 \%)$ showed myopia (from -0.5 to $-4.5 \mathrm{D}$ ), 1 eye $(3.1 \%)$ was hypermetropic $(+1.0 \mathrm{D})$ and 7 eyes $(21.9 \%)$ had the best VA without correction.

The average VA in 7 patients (14 eyes) in whom the first subjective problems developed between the 1030 years of age was 0.45 (from 0.8 to 0.05 ). Their average age during the examination at our department was 30.4 years (ages from 27 to 39 years) and their subjective problems had lasted for 8.7 years on average (from 1 to 29 years).

The average VA in 9 patients (18 eyes) in whom the first subjective problems developed between 31-69 years of age was 0.37 (from 0.8 to 0.025 ). Their average age was 51.9 years (ages from 42 to 73 years) and their subjective problems had lasted for 2.7 years on average (from 1 to 10 years).

Nine patients (18 eyes) had subjective problems lasting for less than 3 years and their average VA was 0.55 (from 0.8 to 0.1 ). Seven patients ( 14 eyes) had subjective problems lasting 3 and more years and their average VA was 0.23 (from 0.8 to 0.025 ).

All the eyes had severely impaired colour sense and 11 patients (68.8\%) perceived the impaired colour sense.

Photophobia troubled 14 patients $(87.5 \%)$ who also reported inferior vision in the daylight. None of our patients suffered from night blindness.

Only 4 eyes had no reduction of the visual field (12.5\%), 5 eyes $(15.6 \%)$ had annular scotoma around the centre, 9 eyes $(28.1 \%)$ had a relative central scotoma and 14 eyes $(43.8 \%)$ had an absolute central scotoma.

Using biomicroscopy and fluorescein angiography, we found changes in RPE, from barely detectable (Fig. 1) to the typical bull's eye (Fig. 2), in all the eyes. We observed non-characteristic slight defects of RPE accompanied by a window effect on FA in 11 eyes (34.4\%), and various degrees of perifoveal atrophy of RPE (bull's eye) in 21 eyes $(65.6 \%)$. The average VA in 11 eyes with noncharacteristic slight defects of RPE was 0.28 (from 0.1 to 0.8 ), the average VA in 21 eyes with bull's eye was 0.48 (from 0.025 to 0.8 ).

All the eyes in the group showed abnormal photopic macular ERG. Twenty two eyes (68.75\%) showed reduced photopic macular ERG response with the average VA of 0.49 (VA from 0.8 to 0.05 ). Ten eyes (31.25\%) showed 


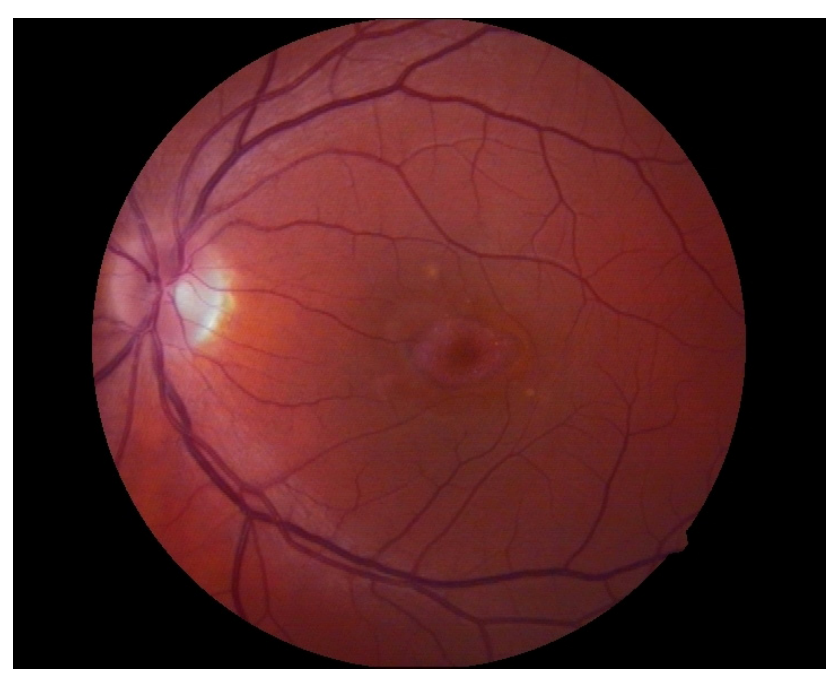

Fig. 1A. Left eye of the patient with the visual acuity of 0.66 , typical image of the bull's eye with distinct perifoveal retinal pigment epithelial atrophy and relatively preserved retinal pigment epithelium in the central macula.

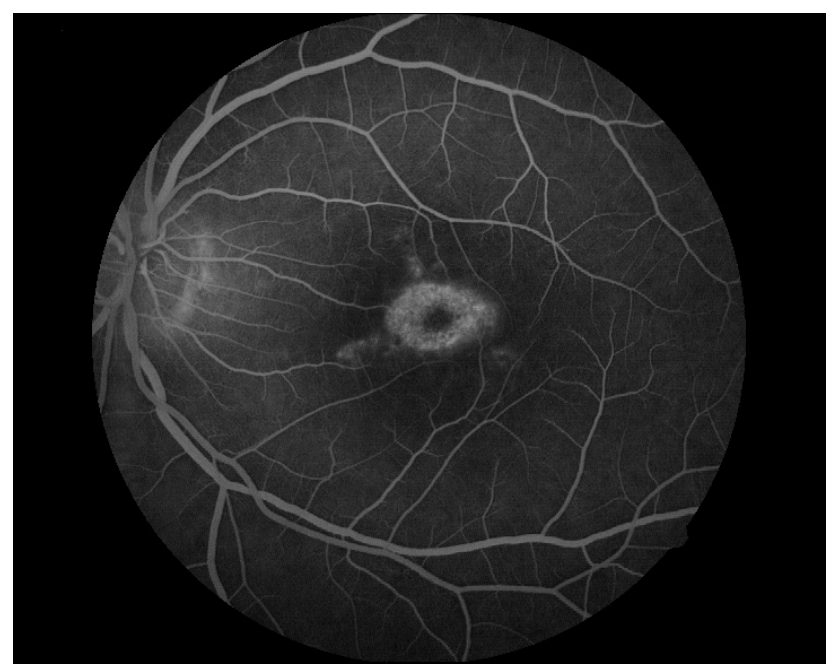

Fig. 1B. Fluorescein angiography, $1^{\text {st }}$ minute. Perifoveal grainy hyperfluorescence of the retinal pigment epithelium defects.

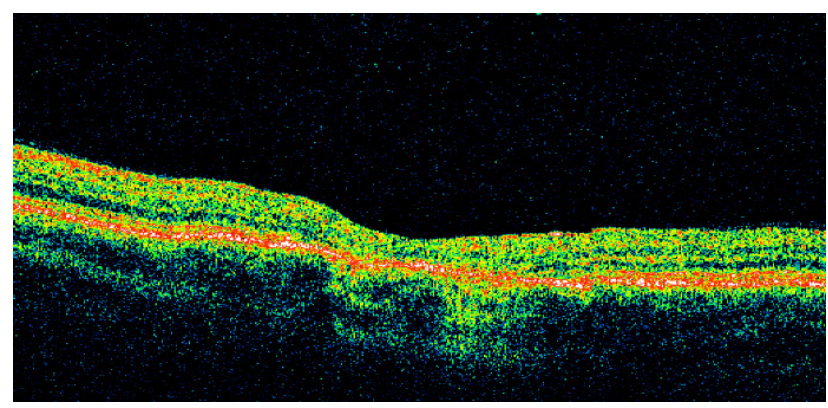

Fig. 1C. Optical coherence tomography: Shallowed foveolar depression resulting from atrophy of the neuroretina. Linearity of the neuroretinal layers is virtually eliminated, especially in the centre of the macula, and the reflectivity is almost homogenously increased. Layers of the photoreceptor inner segment/ outer segment junction and outer nuclear layer are eliminated in the centre of the macula, window effects are visible perifoveally suggesting retinal pigment epithelium atrophy.

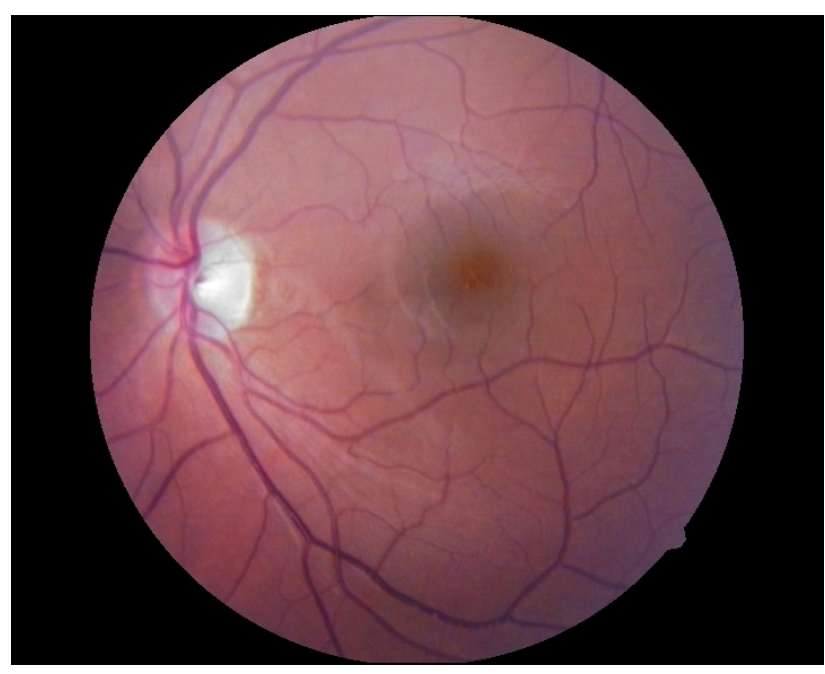

Fig. 2A. Left eye of the patient with the visual acuity of 0.8 with indistinct shifts of pigment in the macula.

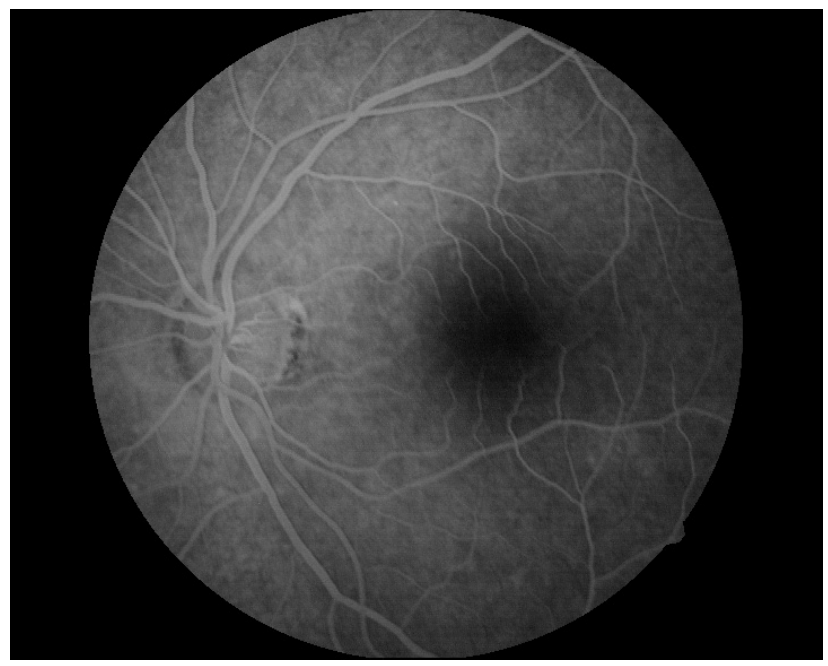

Fig. 2B. Fluorescein angiography, $1^{\text {st }}$ minute. Basic fluorescence of the posterior pole of the eye is in places slightly irregular and represents the fine window effects of small defects of the retinal pigment epithelium.

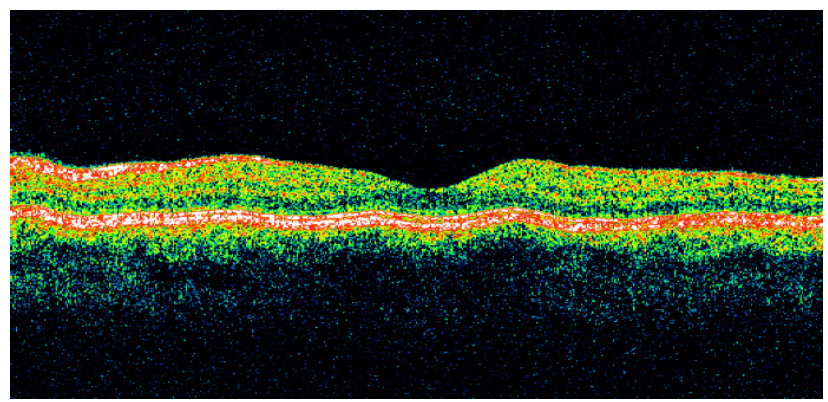

Fig. 2C. Optical coherence tomography: Foveolar depression is visible, the neuroretina is slightly thinned and the linearity of its layers is changed only minimally. Layers corresponding to the photoreceptor inner segment/outer segment junction, outer nuclear layer and retinal pigment epithelium are preserved. 
no response with the average VA of 0.23 (VA from 0.66 to 0.025 ).

Twelve eyes (37.5\%) showed reduced response of the scotopic system while the other eyes $(62.5 \%)$ showed normal response.

OCT displayed reduced thickness and structural changes of the neuroretina in all the eyes. The average thickness of the neuroretina in the centre of the fovea centralis was $86 \pm 31 \mu \mathrm{m}(40-138 \mu \mathrm{m})$ and was the thinnest point of the macula. The neuroretinal thickness was statistically significantly reduced not only in the centre of fovea centralis but also in all the quadrants of the inner and outer sectors (Table 1 ).

In 14 eyes with VA of $0.8-0.5$, the average neuroretinal thickness in the centre of fovea centralis was $91 \pm 24$ $\mu \mathrm{m}$ (from 50 to $130 \mu \mathrm{m}$ ), 11 eyes with VA between 0.4 0.16 had the average neuroretinal thickness in the centre of fovea centralis $97 \pm 32 \mu \mathrm{m}$ (from 50 to $138 \mu \mathrm{m}$ ) and 7 eyes with VA of $0.1-0.025$ had the average neuroretinal thickness in the centre of fovea centralis $61 \pm 23 \mu \mathrm{m}$ (from
40 to $109 \mu \mathrm{m})$. The values of the reduction of neuroretinal thickness in each quadrant of the inner and outer sectors in relation to VA are presented in Table 2.

The normal structure of the macular neuroretina which is characterized by alternation of layers with higher and lower reflectivity was either indistinct or not completely evident in all the eyes of the set. Atrophy was evident especially in the outer nuclear layer (ONL) and at the photoreceptor inner segment/outer segment junction (IS/OS). The outer nuclear layer could not be differentiated in 15 eyes (46.88\%) while in 17 eyes $(53.12 \%)$ it was discernible, however, with irregularly higher reflectivity and an unevenly reduced thickness.

In 16 eyes (50\%), the thin layer of higher reflectivity between the ONL and RPE which corresponds to the IS/ OS was not displayed at all. In 13 eyes (40.63\%), the IS/ OS was discontinuous and in some places it was blended with the adjacent layers. In 3 eyes $(9.37 \%)$, the IS/OS was displayed; in 2 eyes (6.25\%) of one patient, however, we detected a discrete slit-like ablation of the neuroretina

Table 1. Macular thickness measurements of the PCD group, the control group of healthy eyes and the statistical comparison of this groups (average $\pm \mathrm{SD})$.

\begin{tabular}{|c|c|c|c|c|}
\hline \multicolumn{2}{|c|}{$\begin{array}{l}\text { The average neuroretinal thickness } \\
\qquad(\mu \mathrm{m}) \text { in the: }\end{array}$} & \multirow{2}{*}{$\begin{array}{l}\text { The PCD group of } 16 \text { patients } \\
\qquad \begin{array}{l}n=32 \text { eyes } \\
86 \pm 31\end{array}\end{array}$} & \multirow{2}{*}{$\begin{array}{l}\text { The control group of healthy } \\
\text { eyes } 100 \text { individuals } \\
\mathrm{n}=100 \text { eyes }\end{array}$} & \multirow{2}{*}{$\begin{array}{c}\text { Statistical significance } \\
\text { Mann-Whitney Rank } \\
\text { Sum Test } \\
P<0.001\end{array}$} \\
\hline Centre of fovea $c$ & ntralis & & & \\
\hline \multirow{4}{*}{ Inner sector -quadrant } & nasal & $208 \pm 29$ & $285 \pm 14$ & $P<0.001$ \\
\hline & inferior & $214 \pm 29$ & $284 \pm 13$ & $P<0.001$ \\
\hline & superior & $209 \pm 27$ & $286 \pm 14$ & $P<0.001$ \\
\hline & temporal & $197 \pm 27$ & $271 \pm 13$ & $P<0.001$ \\
\hline \multirow{4}{*}{ Outer sector -quadrant } & nasal & $219 \pm 34$ & $268 \pm 16$ & $P<0.001$ \\
\hline & inferior & $196 \pm 34$ & $241 \pm 14$ & $P<0.001$ \\
\hline & superior & $205 \pm 35$ & $248 \pm 14$ & $P<0.001$ \\
\hline & temporal & $180 \pm 33$ & $234 \pm 12$ & $P<0.001$ \\
\hline The average VA & & $0.41 \pm 0.28$ & $1.0 \pm 0.00$ & $P<0.001$ \\
\hline
\end{tabular}

Legend: PCD-the progressive cone dystrophy, VA-the visual aciuty.

Table 2. Macular thickness measurements of the PCD group and the control group of healthy eyes (average \pm SD).

\begin{tabular}{|c|c|c|c|c|c|c|}
\hline \multirow{2}{*}{\multicolumn{2}{|c|}{$\begin{array}{l}\text { The average neuroretinal thickness } \\
\qquad(\mu \mathrm{m}) \text { in the: }\end{array}$}} & \multicolumn{3}{|c|}{ The PCD group with the visual acuity } & \multirow{2}{*}{$\begin{array}{l}\text { The control } \\
\text { group of } \\
\text { healthy eyes } \\
\text { with VA } 1.0 \\
\text { n=100 eyes }\end{array}$} & \multirow{2}{*}{$\begin{array}{c}\text { Statistical } \\
\text { significance } \\
\text { Mann-Whitney } \\
\text { Rank Sum } \\
\text { Test * }\end{array}$} \\
\hline & & $0.8-0.5$ & $0.4-0.16$ & $0.1-0.025$ & & \\
\hline \multicolumn{2}{|l|}{ Centre of fovea centralis } & $91 \pm 24$ & $97 \pm 32$ & $61 \pm 23$ & $160 \pm 11$ & $P<0.001$ \\
\hline \multirow{4}{*}{ Inner sector -quadrant } & nasal & $214 \pm 31$ & $215 \pm 28$ & $187 \pm 14$ & $285 \pm 14$ & $P<0.001$ \\
\hline & inferior & $221 \pm 26$ & $218 \pm 32$ & $191 \pm 16$ & $284 \pm 13$ & $P<0.001$ \\
\hline & superior & $216 \pm 23$ & $216 \pm 29$ & $188 \pm 14$ & $286 \pm 14$ & $P<0.001$ \\
\hline & temporal & $198 \pm 25$ & $204 \pm 31$ & $179 \pm 15$ & $271 \pm 13$ & $P<0.001$ \\
\hline \multirow{4}{*}{ Outer sector -quadrant } & nasal & $227 \pm 34$ & $219 \pm 33$ & $201 \pm 26$ & $268 \pm 16$ & $P<0.001$ \\
\hline & inferior & $203 \pm 31$ & $193 \pm 36$ & $181 \pm 33$ & $241 \pm 14$ & $P<0.001$ \\
\hline & superior & $219 \pm 30$ & $202 \pm 33$ & $184 \pm 31$ & $248 \pm 14$ & $P<0.001$ \\
\hline & temporal & $191 \pm 29$ & $179 \pm 35$ & $159 \pm 30$ & $234 \pm 12$ & $P<0.001$ \\
\hline
\end{tabular}

Legend: PCD-the progressive cone dystrophy, VA-the visual acuity, *-the results are valid for all individual columns of the PCD group. 
Table 3. The IS/OS layer in relation to the neuroretinal thickness, the VA and the shape of the ONL and the RPE.

\begin{tabular}{cccc}
\hline & \multicolumn{2}{c}{ IS/OS layer of the PCD group (n=32 eyes) } \\
\cline { 2 - 4 } & $\begin{array}{c}\text { not evident } \\
\mathrm{n}=16 \text { eyes }\end{array}$ & $\begin{array}{c}\text { discontinuous } \\
\mathrm{n}=13 \text { eyes }\end{array}$ & $\begin{array}{c}\text { continuous } \\
\mathrm{n}=3 \text { eyes }\end{array}$ \\
\hline $\begin{array}{c}\text { The average neuroretinal thickness }(\mu \mathrm{m}) \text { in the } \\
\text { centre of FC: (average } \pm \text { SD) }\end{array}$ & $72 \pm 26$ & $94 \pm 29$ & $0.67 \pm 0.19$ \\
\hline The average VA & $0.29 \pm 0.28$ & $29 \pm 3$ & $0.48 \pm 0.24$ \\
\hline $\begin{array}{c}\text { not evident } \\
\text { (number of eyes) } \\
\text { partly defective } \\
\text { (number of eyes) }\end{array}$ & 13 & 11 & 3 \\
\hline $\begin{array}{c}\text { discontinuous } \\
\text { (number of eyes) } \\
\text { continuous } \\
\text { (number of eyes) }\end{array}$ & 3 & 9 & 2 \\
\hline
\end{tabular}

Legend: IS/OS-the photoreceptor inner segment/outer segment junction, VA-the visual acuity, ONL-the outer nuclear layer, RPE-the retinal pigment epithelium layer, PCD-the progressive cone dystrophy, FC-the fovea centralis

under the fovea centralis. In 26 eyes (81.25\%), the highly reflective layer of the RPE was fragmented to various degree and the areas of the defective RPE displayed the distinct window effect. RPE was observed as a continuous layer in 6 eyes (18.75\%).

In 16 eyes (50\%) without visible IS/OS, the average neuroretinal thickness in the centre of fovea centralis was $72 \pm 26 \mu \mathrm{m}$ (from 40 to $119 \mu \mathrm{m}$ ) and the average VA was $0.29 \pm 0.28$ (from 0.8 to 0.025 ), 13 eyes (40.63\%) with discontinuous IS/OS had the average neuroretinal thickness in the centre of fovea centralis of $94 \pm 29 \mu \mathrm{m}$ (from 50 to $138 \mu \mathrm{m}$ ) and the average VA of $0.48 \pm 0.24$ (from 0.8 to $0.2), 3$ eyes $(9.37 \%)$ with the continuous IS/OS had the average neuroretinal thickness in the centre of fovea centralis of $129 \pm 3 \mu \mathrm{m}$ (from 125 to $132 \mu \mathrm{m}$ ) and the average VA of $0.67 \pm 0.19$ (Table 3 ).

\section{DISCUSSION}

OCT, which displays a sectional view of the macula, provided new insight into PCD. Emfietzoglou et al. ${ }^{17}$ observed in one patient ablation of thinned neuroretina and a cystoid oedema in the macula. Wolfing et al. ${ }^{16}$ described one patient and Lim et al. ${ }^{15}$ described two patients with dystrophy of cones and rods. They observed reduced retinal thickness in the fovea centralis and they localized the reduced thickness predominantly to its outer layers In agreement with them we observed reduced neuoretinal thickness in the fovea centralis and macula (vs. healthy eyes) in all our patients. The average neuroretinal thickness of our group's eyes manually measured in the fovea centralis centre $(86 \pm 31 \mu \mathrm{m})$ was markedly lower than the manually measured value in our control group of 100 healthy eyes $(160 \pm 11 \mu \mathrm{m})$ and the 37 healthy eyes $(170 \pm 18$ $\mu \mathrm{m})$ reported by Chan et al. ${ }^{18}$. In some eyes with markedly reduced neural retinal thickness in the fovea centralis, it was impossible to obtain an accurate demarcation of the fovea centre with a radius of $0.5 \mathrm{~mm}$ by the OCT software. Similarly, average neuroretinal thickness in the perifoveal sectors with radii of 0.5-1.5 and 1.5-3.0 $\mathrm{mm}$ which was measured by the OCT software was lower than that of healthy eyes and the values were statistically significant $(P<0.001)$ (Table 1). Unlike Wolfing et al. ${ }^{16}$, who found normal retinal thickness of the nasal perifoveal region, in our patients we observed a reduced retinal thickness in this region as well. The neuroretinal thickness in the fovea centralis centre and the adjacent macular sectors was not the only determining factor for VA. Above all, VA was dependent on how well the IS/OS continuity was preserved and, to a lesser degree, on the image of the ONL and RPE layers. Eyes with better preserved neuroretinal structure in the fovea centralis had mostly less reduced retinal thickness and better VA.

Stratus OCT is the last generation in the development of Carl Zeiss Meditec time domain optical coherence tomography. Recently, images of intraretinal changes in Stargardt's macular dystrophy and adult viteliform macular dystrophy have been obtained using ultrahigh resolution optical coherence tomography with a resolution of $3 \mu \mathrm{m}$ in the axial direction ${ }^{19,20}$. Lim et al. ${ }^{15}$ examined 2 patients with cone-rod dystrophy using a high-speed, high resolution, Fourier-domain OCT, which is already commercially available, with an axial resolution of $5 \mu \mathrm{m}$. It can be assumed that these new devices will provide even more detailed information about structural changes in the neuroretina in PCD thus contributing to better insight into this condition.

We showed RPE abnormalities by ophthalmoscopy, biomicroscopy and fluorescein angiography, however, their extent did not always correspond to the loss of VA. In some patients we found relatively good VA with the finding of "bull's eye" while less obvious and non-characteristic changes in RPE were sometimes accompanied by severe loss of VA. VA is determined by the number of preserved cones in the fovea centralis. In the case of "bull's 
eye", the highest loss of cones and subsequently also RPE cells can be expected in the perifoveal region while relatively good VA may be maintained for a long time.

ERG results confirmed benefits of this test for PCD. Photopic macular ERG response was always subnormal or extinguished and it usually characterized the loss of VA. Only in one patient, as an exception, we observed an extinguished photopic macular ERG response at VA of 0.66 .

Our PCD patients represented only a small fraction $(0.00025 \%)$ of the total number of patients examined at our clinic over two years.

With such a rare disease, this number was not insignificant and a more frequent diagnosis of PCD can be expected in outpatient practice as long as the PCD is considered in the differential diagnosis and if up-to-date examination procedures are applied.

Our findings confirmed the wide spectrum of clinical pictures of PCD. Heritability was confirmed in nearly one third of the patients $(31.25 \%)$, members of the two families. In one family, with mother, son and daughter affected, heritability was autosomally dominant, in the other family with the occurrence in the brother and sister, most probably autosomally recessive heritability was involved. However, autosomally recessive heritability cannot be ruled out in the other patients with sporadic occurrence.

Worthy of attention are data on the onset of the disease. Sadowski and Zrenner ${ }^{1}$ recorded the onset of the condition based on the first subjective problems within the first 30 years of life in all 40 patients with PCD. In our group, the onset of visual problems after reaching 30 years of age was reported by more than half of the patients (56.25\%) while the latest onset, at the age of 69 years, was reported by the oldest patient who was the mother of the two affected children. There may be significant individual differences in the perception of slowly progressing deterioration of visual functions which are determined by intellect as well as varying degree of demand for the quality of visual functions. This is illustrated by the oldest patient who was diagnosed with PCD in connection with the condition in her children, and not until reaching a fairly advanced stage of progressions.

\section{CONCLUSION}

It can be concluded that PCD incidence is most likely higher than assumed. The spectrum of clinical pictures of PCD, which is often heritable, is wide and non-characteristic in some patients, and barely detectable findings in the macula may be a source of diagnostic difficulties. OCT specifies the quantitative and qualitative changes in the macula and can significantly contribute to diagnosing PCD, especially in the early stages of the condition where it can be difficult to diagnose.

\section{ABBREVIATIONS}

PCD, The progressive cone dystrophy; VA, The visual acuity; RPE, The retinal pigment epithelium; OCT, The optical coherence tomography; FA, The fluorescein angiography; ONL, The outer nuclear layer; IS/OS, The photoreceptor inner segment/outer segment junction.

\section{ACKNOWLEDGEMENT}

Autorship contributions: JZ: literature search , manuscript writing, study design, data collection, analysis and interpretation, statistical analysis, figures, final approval; JL: data analysis of electrophysiological tests; IK: manuscript writing, study design, data analysis and interpretation, final approval.

Conflict of interest statement: none declared.

\section{REFERENCES}

1. Sadowski B, Zrenner E. Cone and rod function in cone degenerations. Vision Res 1997;37:2303-14.

2. Simunovic MP, Moore AT. The cone dystrophies. Eye 1998;12:553-65.

3. Krill AE, Deutman AF, Fishman M. The cone degenerations. Doc Ophthalmol 1973;35:1-80.

4. Michaelides M, Aligianis IA, Ainsworth JR, Good P, Mollon JD, Maher ER, Moore AT, Hunt DM. Progressive cone dystrophy associated with mutation in CNGB3. Invest Ophthalmol Vis Sci 2004;45:1975-82.

5. Michaelides M, Wilkie SE, Jenkins S, Holder GE, Hunt DM, Moore AT, Webster AR. Mutation in the gene GUCA1A, encoding guanylate cyclase-activating protein 1, causes cone, cone-rod, and macular dystrophy. Ophthalmology 2005;112:1442-7.

6. Michaelides M, Hardcastle AJ, Hunt DM, Moore AT. Progressive cone a cone-rod dystrophies: phenotypes and underlying molecular genetic basis. Surv Ophthalmol 2006;51:232-58.

7. Carr RE. Cone dystrophies. In: Guyer DR, Yannuzzi LA, Chang S, Shields JA, Green WR. Retina-Vitreous-Macula. Philadelphia, London, Toronto, Montreal, Sydney, Tokyo: W.B. Saunders Comp; 1999.p.9428

8. Heckenlively JR, Weleber RG. X-linked recessive cone dystrophy with tapetal-like sheen. A newly recognized entity with Mizuo-Nakamura phenomenon. Arch Ophthalmol 1986;104:1322-8.

9. Downes SM, Holder GE, Fitzke FW, Payne AM, Warren MJ, Bhattacharya SS, Bird AC. Autosomal dominant cone and conerod dystrophy with mutations in the guanylate cyclase activator $1 \mathrm{~A}$ gene-encoding guanylate cyclase activating protein-1. Arch Ophthalmol 2001;119:96-105.

10. Meire FM, Bergen AA, De Rouck A, Leys M, Delleman JW. X linked progressive cone dystrophy. Localisation of the gene locus to Xp21-p11.1 by linkage analysis. Br J Ophthalmol 1994;78:103-8.

11. Ripps H, Noble KG, Greenstein VC, Siegel IM, Carr RE. Progressive cone dystrophy. Ophthalmology 1987;94:1401-9.

12. Jacobson DM, Thompson HS, Bartley JA. X-linked progressive cone dystrophy. Clinical characteristics of affected males and female carriers. Ophthalmology 1989;96:885-95.

13. Scheiner EM. Progressive cone-rod dystrophy. Clin Eye Vis Care 1999;10:173-9.

14. Francois J, De Rouck A, De Laey JJ. Progressive cone dystrophies. Ophthalmologica 1976;173:81-101.

15. Lim Jl, Tan O, Fawzi AA, Hopkins JJ, Gil-Flamer JH, Huang D. A pilot study of Fourier-domain optical coherence tomography of retinal dystrophy patients. Am J Ophthalmol 2008;146:417-26.

16. Wolfing Jl, Chung M, Carroll J, Roorda A, Williams DR. Highresolution retinal imaging of cone-rod dystrophy. Ophthalmology 2006;113:1014-9. 
17. Emfietzoglou I, Grigoropoulos V, Nikolaidis P, Theodossiadis G, Rouvas, Theodossiadis P. Optical coherence tomography findings in a case of cone-rod dystrophy. Ophthalmic Surg Lasers Imaging 2010;41:e1-3. doi:10.3928/15428877-20101124-10

18. Chan A, Duker JS, Ko TH, Fujimoto JG, Schuman JS. Normal macular thickness measurements in healthy eyes using Stratus optical coherence tomography. Arch Ophthalmol 2006;124:193-8.
19. Wirtitsch MG, Ergun E, Hermann B, Unterhuber A, Stur M, Scholda C, Sattmann H, Ko TH, Fujimoto JG, Drexler W. Ultrahigh resolution optical coherence tomography in macular dystrophy. Am J Ophthalmol 2005;140:976-83.

20. Srinivasan VJ, Wojtkowski M, Witkin AJ, Duker JS, Ko TH, Carvalho M, Schuman JS, Kowalczyk A, Fujimoto JG. High-definition and 3-dimensional imaging of macular pathologies with high-speed ultrahigh-resolution optical coherence tomography. Ophthalmology 2006;113:2054-65. 